\title{
Effects of frequency mismatch and wave dispersion on a self-consistent Hamiltonian model for an arbitrary-amplitude cyclotron-resonance laser accelerator
}

\author{
R. Pakter, R. S. Schneider, and F. B. Rizzato \\ Instituto de Física, Universidade Federal do Rio Grande do Sul, Caixa Postal 15051, \\ 91501-970 Porto Alegre, Rio Grande do Sul, Brazil
}

(Received 4 March 1993; revised manuscript received 23 August 1993)

\begin{abstract}
In this work, we develop a self-consistent, nonperturbative Hamiltonian formulation for the cyclotron-resonance laser accelerator. The formalism takes into account wave dispersion and frequency mismatch, enabling one to show how the mismatch can be used to remove some of the energization limits imposed by dispersion.

PACS number(s): 41.75.Ht, 41.60.-m
\end{abstract}

\section{INTRODUCTION}

A promising configuration for laser acceleration is the cyclotron-resonance laser accelerator (CRLA) [1], where a coherent electromagnetic wave may transfer a large amount of energy to a beam of electrons gyrating in a guide magnetic field. This large amount of transferred energy takes place because of the autoresonance mechanism [2,3] where, under some ideal conditions, an initial wave-particle synchronism is self-sustained throughout the accelerating period.

It has been observed, however, that one of these CRLA ideal conditions is hardly obtained in feasible experimental schemes. This particular condition is the one requiring the laser field to be dispersionless [4], a very restrictive condition if one takes into account dispersive effects arising from the confining wave guides [5]. The analysis of dispersive CRLA's has been recently carried out both analytically (in a perturbative fashion) [6] and numerically [4], where it was shown that a typically small degree of dispersion may create some severe limits to the accelerating efficiency. On the other hand, for the unlikely situation of extremely small dispersion and equally small wave amplitudes, the accelerating efficiency was shown to be larger than in the previous case, turning out to be critically dependent on the total amount of free electromagnetic energy. In this latter case, one cannot simply solve the relativistic particle equations under the influence of a given electromagnetic wave; one has to consider the selfconsistent dynamics of particle plus waves.

In the present work we intend to investigate further the mentioned self-consistent wave-particle interaction. To this end, we develop a general formalism where under the macroparticle approximation, both particle and wave dynamics can be canonically obtained from one generalized Hamiltonian. The formalism is general in two basic senses: it is not perturbative in wave amplitudes [7], and it allows for the inclusion of a frequency mismatch $\delta=\omega-\left(\omega_{c 0} / \gamma+k v_{z}\right) \ll \omega$, where $\omega$ is the wave frequency, $k$ is the wave vector, $\omega_{c 0}$ is the nonrelativistic cyclotron frequency, $v_{z, 0}$ is the initial longitudinal velocity, and $\gamma_{0}$ is the initial value of the relativistic factor (henceforth, subindex " 0 " denotes initial value). Magnetic field tape ring is not included, but by varying the mismatch, we shall show how to compensate and even partially eliminate the limiting effects due to dispersion on acceleration processes. The formalism will be applied in the microwave range (frequencies on the order of $\mathrm{GHz}$ ), where we shall see that even if one considers dispersive systems, wave dynamics may be an important factor to take into consideration in view of the fact that careful choices of the mismatch can cause the particles to absorb an amount of energy comparable to that contained in the initial laser field.

This paper is organized as follows. In Sec. II we introduce the model and briefly derive the complete set of wave-particle dynamical equations; in Sec. III, using the macroparticle approximation, we obtain a self-consistent Hamiltonian formalism governing the dynamics; in Sec. IV we perform a numerical investigation on the analytical results along with a simulation analysis to check the validity of the macroparticle approach, and in Sec. V we conclude the work.

\section{MODEL AND BASIC EQUATIONS}

Let us consider an electron beam and a circularly polarized electromagnetic wave, copropagating along the homogeneously magnetized $\mathbf{z}$ axis of the chosen reference frame.

The circularly polarized wave vector potential is written as

$$
\frac{e}{m c^{2}} \mathbf{A} \equiv-\frac{1}{2} \sqrt{\rho} e^{i \sigma} e^{i \hat{\omega}(f z-t)} \widehat{\mathbf{e}}_{c}+\text { c.c. },
$$

where $\rho$ and $\sigma$ have a slow-time dependence, $e$ is the electron charge, $\widehat{\mathbf{e}}_{c} \equiv \hat{\mathbf{x}}+i \hat{\mathbf{y}}$, and $\hat{\omega} \equiv \omega / \omega_{c 0}$. The wave frequency $\omega$ satisfies a dispersion relation of the form $\omega=\omega(k, f)$ where $\omega(k, f)$ is modeled as $\omega(k, f) / c k \equiv f^{-1}$ $(\geq 1.0)$. Factor $f$ accounts for wave dispersion (it could be a factor connected with the finite transverse dimensions of some guiding system) and it is related to the dispersion factor $\beta_{\mathrm{ph}}$ of Ref. [4] through $f=\beta_{\mathrm{ph}}^{-1}$. We take $k$ as the wave vector, $\omega_{c 0} \equiv\left|e B_{z 0} / m c\right|$ with $B_{z 0}$ as the background magnetic field, and normalize time and space to $\omega_{c 0}$ and $\omega_{c 0} / c$, respectively. Then it becomes 
possible to cast the Hamiltonian that governs the dynamics of a single electron in the form

$$
\begin{aligned}
\mathcal{H}= & \left(1+\left\{P_{x}+\sqrt{\rho} \cos [\widehat{\omega}(f z-t)+\sigma]\right\}^{2}\right. \\
& \left.+\left\{x+P_{y}-\sqrt{\rho} \sin [\widehat{\omega}(f z-t)+\sigma]\right\}^{2}+P_{z}^{2}\right)^{1 / 2},
\end{aligned}
$$

with $\mathscr{H} / m c^{2} \rightarrow \mathscr{H}$ and $\mathbf{P} / m c \rightarrow \mathbf{P}$.

The structure of the Hamiltonian can be made simpler if one introduces canonical guiding-center variables $I$ and $\phi$ through $P_{x}=\sqrt{2 I} \cos \phi$ and $x+P_{y}=\sqrt{2 I} \sin \phi$ along with the additional canonical transformations $\phi+\hat{\omega}(f z-t) \rightarrow \phi, P_{z} \rightarrow P_{z}+f \hat{\omega} I$, and $\mathscr{H} \rightarrow \mathscr{H}+\hat{\omega} I$. This enables us to rewrite the Hamiltonian (2) as

$$
\mathscr{H}=-\hat{\omega} I+\sqrt{1+2 I+\left(P_{z}+\hat{\omega} f I\right)^{2}+2 \sqrt{2 I} \rho \cos (\phi+\sigma)+\rho} \equiv-\hat{\omega} I+\Gamma .
$$

The slow-time self-consistent evolution equation for the amplitude of the vector potential is readily derived from Maxwell's equations in dimensional form

$$
i \omega d_{t}\left(\sqrt{\rho} e^{i \sigma}\right)=\frac{4 \pi e}{m c V T} \int \mathrm{J}_{\perp} \cdot \hat{\mathrm{e}}_{c}^{*} e^{-i\left(k z^{\prime}-\omega t^{\prime}\right)} d^{3} \mathbf{r}^{\prime} d t^{\prime}
$$

$\left(d_{t} \equiv d / d t\right)$, where we have performed a Fourier transform over the fast (primed) variables introducing the volume $V$ and the period $T$. The current density on the $(x, y)$ plane is written as

$$
\mathbf{J}_{\perp}=\sum_{i} e \mathbf{v}_{\perp, i}\left(t^{\prime}\right) \delta\left(\mathbf{r}^{\prime}-\mathbf{r}_{i}\left(t^{\prime}\right)\right)
$$

with the subscript $i=1,2, \ldots, N$ labeling the $N$ particles present in the system; $\mathbf{v}_{1, i}\left(t^{\prime}\right)$ denotes the perpendicular component of their velocities and $\mathbf{r}_{i}\left(t^{\prime}\right)$ represents their instantaneous positions. Now, one writes the canonical connection between velocity and momentum

$$
\Gamma_{i} \mathbf{v}_{\perp, i}=\mathbf{P}_{1, i}-\frac{e \mathbf{A}}{m c^{2}}+x_{i} \hat{\mathbf{y}},
$$

where adimensionalization is reintroduced. With relations (5) and (6) and taking into account the approximate resonance condition

$$
\omega \sim \frac{\omega_{c 0}}{\Gamma_{i}}+k v_{z, i}
$$

it becomes possible to cast Eq. (4) in the form

$$
i d_{t}\left(\sqrt{\rho} e^{i \sigma}\right)=\frac{2 \pi e^{2}}{m \omega \omega_{c 0} V} \sum_{i}\left[\frac{\sqrt{2 I_{i}}}{\Gamma_{i}} e^{-i\left\{\phi_{i}(t)+\widehat{\omega}\left[f z_{i}(t)-t\right]\right\}}+\frac{\sqrt{\rho} e^{i \sigma}}{\Gamma_{i}}\right],
$$

with the resonant wave-particle phase $\phi_{i}+\hat{\omega}\left(f z_{i}-t\right)$ as a variable depending only on the slow-time scales.

Then, if one identifies the factor $\phi_{i}(t)+\hat{\omega}\left[f z_{i}(t)-t\right]$ of Eq. (7) as the canonically transformed wave-particle phase factor introduced in Eq. (3), it becomes possible to write the complete set of wave-particle dynamical equations in the form

$$
\begin{aligned}
& d_{t} I_{i}=-\partial_{\phi_{i}} \mathcal{H}_{i}=\frac{\sqrt{2 I_{i} \rho}}{\Gamma_{i}} \sin \left(\phi_{i}-\sigma\right), \\
& d_{t} \phi_{i}=\partial_{I_{i}} \mathcal{H}_{i}=-\hat{\omega}+\frac{\left.1+\hat{\omega} f\left(P_{z, i}+\hat{\omega} f I_{i}\right)+\sqrt{\rho /\left(2 I_{i}\right.}\right) \cos \left(\phi_{i}-\sigma\right)}{\Gamma_{i}}, \\
& d_{t} \rho=-\left\langle\frac{\lambda \sqrt{2 I_{i} \rho}}{\Gamma_{i}} \sin \left(\phi_{i}-\sigma\right)\right\rangle,
\end{aligned}
$$

and

$$
d_{t} \sigma=\left\langle\frac{\lambda \sqrt{2 I_{i}}}{2 \Gamma_{i}} \frac{1}{\sqrt{\rho}} \cos \left(\phi_{i}-\sigma\right)+\frac{\lambda}{2 \Gamma_{i}}\right\rangle,
$$

where $\langle G(I, \phi)\rangle \equiv \Sigma_{i} G\left(I_{i}, \phi_{i}\right) / N$ for a generic oneparticle function $G(I, \phi)$, and where we define $\lambda \equiv \omega_{p}^{2} /\left(\omega \omega_{c 0}\right) \equiv 4 \pi e^{2} N /\left(m \omega \omega_{c 0} V\right) ; \omega_{p}$ plays the role of an average plasma frequency.
The set (8)-(11) can be numerically solved, but before that, let us obtain some analytical information by means of the macroparticle approximation.

\section{MACROPARTICLE APPROXIMATION AND HAMILTONIAN FORMALISM}

The macroparticle approach consists of regarding all the particles as a single entity and its accuracy is closely 
related to the factor $1 / \sqrt{I_{i}}$ present in Eq. (9). Indeed, if the initial value of $I_{i}$ is the same for all particles (let us call it $I_{0}$ ) and is small enough, the mentioned factor is large and drives a fast bunching that causes all the particles to concentrate around the phase space point characterized by $\phi-\sigma=\pi / 2$ and $I=I_{0}$, during the very initial dynamical stages with the subsequent motion being therefore approximately the same for all the particles, no matter how diffuse the initial gyrophase phases were. This hypothesis shall be tested in the next section, but for now let us assume that it is fulfilled.

If this is the case, the particle index $i$ is unnecessary and it is seen immediately that the dynamical equations take the form

$$
\begin{aligned}
& d_{t} I=-\partial_{\phi} \mathcal{H}, \quad d_{t} \phi=\partial_{I} \mathcal{H}, \\
& d_{t} \rho=-2 \lambda \frac{\sqrt{2 I}}{2 \Gamma} \sqrt{\rho} \sin (\phi+\sigma),
\end{aligned}
$$

and

$$
-d_{t} \sigma=\delta+\lambda \frac{\sqrt{2 I}}{2 \Gamma} \frac{1}{\sqrt{\rho}} \cos (\phi+\sigma)+\frac{\lambda}{2 \Gamma} .
$$

Now, if one rescales $\rho$ according to $\rho=\lambda \hat{\rho}$ the interesting and final conclusion is that all the relevant dynamical equations for both particles and fields can be derived from one generalized Hamiltonian given by

$d_{t} I=-\partial_{\phi} \mathfrak{E}, \quad d_{t} \phi=\partial_{I} \mathfrak{S}$,

$d_{t} \rho=-\partial_{\sigma^{\prime}} \mathfrak{s}, \quad d_{t} \sigma^{\prime}=\partial_{\rho} \mathfrak{S}$,

$\mathfrak{5}=-\hat{\omega} I+\Gamma$,

$\Gamma=\left[1+2 I+\left(P_{z}+\hat{\omega} f I\right)^{2}+2 \sqrt{2 I \lambda \hat{\rho}} \cos \left(\phi-\sigma^{\prime}\right)+\lambda \hat{\rho}\right]^{1 / 2}$,

where besides the canonical pair $(I, \phi)$ one considers $\hat{\rho}$ as the "momentum" corresponding to the wave field and $\sigma^{\prime}(=-\sigma)$ as the canonically conjugated coordinate. The canonical transformations $\phi-\sigma^{\prime}=\phi^{\prime}$ and $\hat{\rho}=\rho_{c}-I$ reduce the degrees of freedom ( $\rho_{c}$ is a constant of motion) and allow us to write a final effective canonical system as

$$
\begin{aligned}
& d_{t} I=-\partial_{\phi} \mathfrak{S}, \quad d_{t} \phi=\partial_{I} \mathfrak{H}, \\
& \Gamma=\left[1+2 I+\left(P_{z}+\hat{\omega} f I\right)^{2}+2 \sqrt{2 I \lambda\left(\rho_{c}-I\right)} \cos \phi+\lambda\left(\rho_{c}-I\right)\right]^{1 / 2},
\end{aligned}
$$

where for simplicity the prime has been dropped off the angular variable $\phi$.

Taking into consideration that the above Hamiltonian is time independent, one can derive the relationship

$$
\widehat{\omega} d_{t} I=d_{t} \Gamma,
$$

which can be combined with $d_{t} \hat{\rho}=-d_{t} I$ to furnish

$$
d_{t}(\hat{\omega} \hat{\rho})+d_{t} \Gamma=0,
$$

a relation that indicates energy conservation between wave and particles. We quote that $\hat{\omega} \hat{\rho}_{0} m c^{2}=\omega^{2} \rho_{c} m c^{2}$ may be viewed as the total electromagnetic energy available per particle.

In order to investigate the influence of wave dynamics, wave dispersion, and frequency mismatch on the autoresonance process, let us first recall that according to the simplest definition, autoresonance takes place when we set $f=1, \rho_{c}-I \rightarrow \rho_{c}, \lambda \rho_{c}<1$, and $P_{z}=0$ in Eqs. (19) with $\phi(t=0)=(2 m+1 / 2) \pi$, which means an approximation of small amplitude dispersionless laser waves, energizing both the transverse and longitudinal particle dy- namics (recall that $P_{z}=0$ causes the physical longitudinal momentum to be proportional to $I$ ). Under such circumstances, the canonical equations for $I$ and $\phi$ imply the constancy of the angular variable and the unlimited growth of the momentum $I$. On the other hand, when wave dynamics is taken into account with $f=1$ and the previous initial condition for $\phi$, the canonical equations indicate that the energization process proceeds without variations of $\phi(t)$, up to a maximum value for $I$, readily evaluated as $I_{\max }=\rho_{c}$. Autoresonance is rapidly destroyed for $f<1$ and the corresponding energization is highly reduced. In general, even for lasers of large amplitudes it can be observed that autoresonance is present as long as one takes $f=1$ in the absence of wave dynamics.

To evaluate $I_{\max }$ in the generic situation of $f<1$ and arbitrary amplitudes, it is convenient to combine our canonical equations (19) to obtain a closed equation for $I$ in the form

$$
(\dot{I})^{2}+V(I)=0 .
$$

In the equation above, $V(I)$ is an effective potential driving $I$ excursions, written as

$$
V(I)=-\frac{8 \lambda I\left(\rho_{c}-I\right)-\left\{(h+\widehat{\omega} I)^{2}-\left[1+2 I+(\hat{\omega} f I)^{2}+\lambda\left(\rho_{c}-I\right)\right]\right\}^{2}}{4(h+\hat{\omega} I)^{2}},
$$

where we set $P_{z}=0$ and $h=\mathfrak{F}\left(I_{0}, \phi_{0}\right)$, with $h$ as the numerical (and constant) value of $\mathfrak{S}$ calculated for some initial conditions $I_{0}, \phi_{0} . I_{\max }$ shall be sought as the first nontrivial zero of the function $V(I)$, assuming $\sin \phi_{0}=+1.0$ and $I_{0}=0.0$.

\section{NUMERICAL ANALYSIS}

\section{A. Macroparticle approach}

Now we make a numerical application of the formalism to some experimentally relevant situations. Among 
these situations, we chose to focus on systems operating with electromagnetic modes in the microwave range because this range appears to require the most feasible experimental conditions [4]. Other ranges, such as the optical one, should not be discarded but will not be further investigated here.

Recalling that we are assuming a small mismatch $\delta$ between wave and Doppler-shifted initial cyclotronic frequencies, we write $\omega=\omega_{c 0} / \Gamma_{0}+k v_{z, 0}+\delta$ in dimensional form or

$$
\widehat{\omega}=1 / \Gamma_{0}+\widehat{\omega} f v_{z, 0}+\delta
$$

in the respective adimensional form (with the latter $\delta$ adimensionalized). Considering beams of small initial energies $I_{0}=v_{z, 0}=0.0$, in Fig. 1 we plot $I_{\max }$ vs $\delta$ for different values of the dispersive factor $f$. We take $\omega \approx 10 \mathrm{GHz}$ and the plasma frequency $\omega_{p} \sim 1.0 \mathrm{GHz}$ (tenuous beams of densities $\sim 10^{9} \mathrm{~cm}^{-3}$, which implies $\lambda=0.01$, considering $\rho_{c}=10.0$ in Fig. 1(a), and $\rho_{c}=100.0$ in Fig. 1(b). With these figures one can appreciate the role of the mismatch; while for $f=1.0$ the largest $I$ excursion is attained for $\delta=0.0$, for $f<1$ the largest excursions occur for $\delta<0$.

For nonperturbatively large values of the laser amplitude as in Fig. 1(b) the effects of the $\delta$ and $f$ factors are even sharper. If one does not select a convenient negative $\delta$ one reaches amplitudes much smaller than the max-
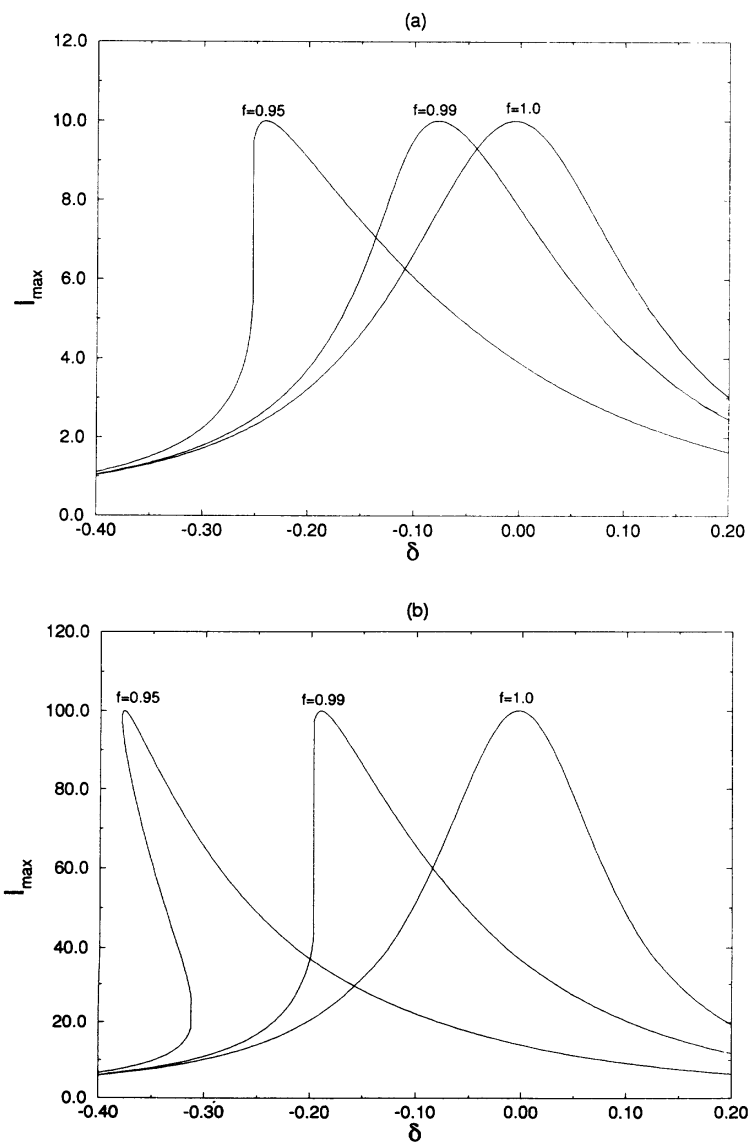

FIG. 1. $I_{\max }$ vs $\delta$ for $\lambda=0.01, I_{0}=0.0, \sin \phi_{0}=1.0$, and various values of $f$. $\rho_{c}=10.0(\mathrm{a})$ and $\rho_{c}=100.0(\mathrm{~b})$. imum allowed. However, with the proper choice, one can partially remove the limiting effects of dispersion and reach the maximum allowed $I_{\max },\left(I_{\max }\right)_{\max }$. It is also seen from Fig. 1(b) that maximum energy exchange does not always occur. If $\rho$ or $f^{-1}(f=0.95$ corresponds to $\left.1-\beta_{\mathrm{ph}} \lesssim 10^{-1}\right)$ is too large, $\left(I_{\max }\right)_{\max }=\rho_{c}$ may not be accessible from small values of $I_{0}$, similarly to what takes place in mismatched beat-wave accelerators.

\section{B. Integration of the complete set of equations}

The calculations carried out so far assume $I_{0}=0.0$, the ideal initial condition for phase bunching. An important issue would be to discuss what happens with the system as one considers larger (and more realistic) values of the initial action. In order to briefly address this point, in Fig. 2 we compare the results of Fig. 1(b) with numerical simulations based on Eqs. (8)-(11). The simulations are performed with $N=500$ particles starting with gyrophases uniformly distributed over $0<\phi<2 \pi$ and with $I_{0}=0.001$ (circles) and $I_{0}=0.5$ (squares); the remaining parameters are the same as used in the macroparticle calculation. We plot the average $\langle I\rangle_{\max }$, to see that the macroparticle model is expected to be adequate only for small values of the initial action. As soon as one increases $I_{0}$, the agreement becomes poorer. Besides, the differences between simulations and macroparticle calculations increase as $-\delta$ gets larger. For these large values of $-\delta$, the maximum $\langle I\rangle$ excursion may be larger in the absence of bunching; however, beam quality is greatly reduced as shall be seen next.

In Fig. 3 the phase space obtained from simulation is plotted for $\delta=-0.1$ and $f=0.99$. In Fig. 3(a) we take $I_{0}=0.001, t=290.0$ and in Fig. 3(b) $I_{0}=0.5, t=180.0$. It becomes clear that a large energy spread does occur for too large values of the initial action.

\section{FINAL REMARKS}

The possibility of reaching maximum allowed $I$ values makes it necessary to include wave dynamics (and its

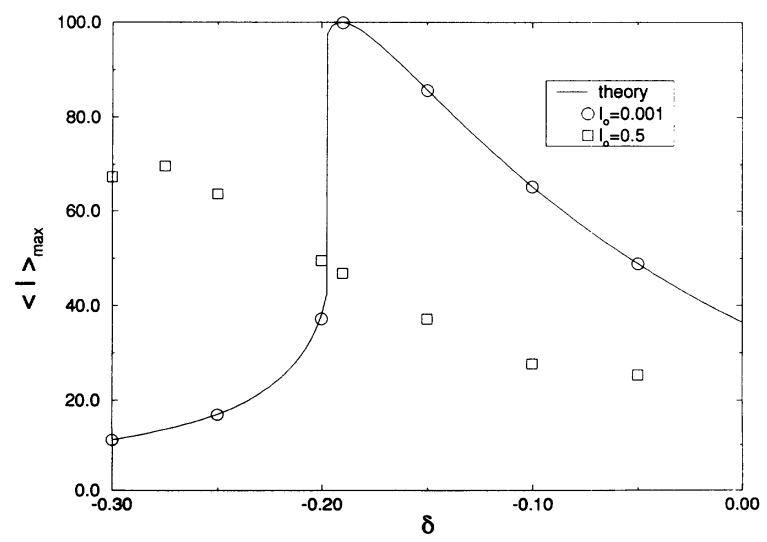

FIG. 2. Comparison of the macroparticle model of Fig. 1(b) with $f=0.99$, with the corresponding numerical simulations. The simulations are obtained for $I_{0}=0.001$ (circles) and $I_{0}=0.5$ (squares). 

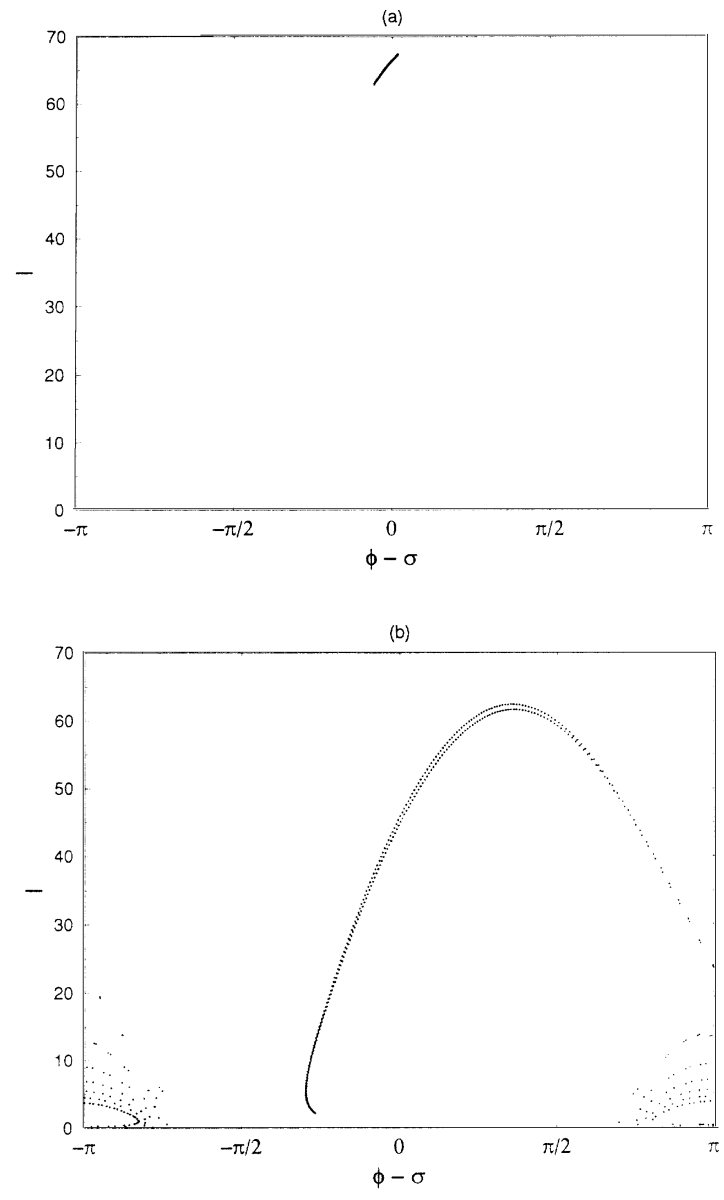

FIG. 3. Phase spaces generated from simulation. We consider $f=0.99, \delta=-0.1$. In (a) $I_{0}=0.001$ and $t=290.0$; in (b) $I_{0}=0.5$ and $t=180.0$.

finite amount of free energy) in the formalism. Other effects such as synchrotron emission and beam defocusing or beam spread also limit the potential for maximum wave-particle energy exchange interaction, saturating the process even within the macroparticle approximation [7]. Synchrotron emission, however, is important for particles with energies on the order of $\mathrm{TeV}$ [8], which are much larger than the ones acquired by particles in the microwave range. For instance, even in the cases where $\left(I_{\max }\right)_{\max }=\rho_{c}=100.0$, the maximum kinetic energy attained is approximately given by $E_{\max }=\left(\Gamma_{\max }-1\right) m c^{2}$
$=I_{\max } m c^{2} \lesssim 0.1 \mathrm{GeV}$ if $m c^{2} \lesssim 1 \mathrm{MeV}$. As for spread of the particle beam one can start by estimating the particle gyroradius as $r \sim\left(c / \omega_{c 0}\right) \sqrt{2 I}$. For some typical frequencies on the order $10-100 \mathrm{GHz}$ and even for the nonperturbatively maximum gain of Fig. 1 (b) $I=\left(I_{\max }\right)_{\max } \sim \rho_{c}$, this yields $r \sim 1-10 \mathrm{~cm}$ for $\lambda \sim 0.01$, which are radii not much larger than the initial beam radius itself; any increase on the magnetic field strength (with a subsequent increase on the resonant frequency) or any reduction on $\rho_{c}$ can further reduce the gyroradius and the spread.

If one notes that the slow-time scale is regulated by the parameter $\lambda$, the interaction time interval $t_{\max }$ for maximum energy extraction may be estimated as $\omega t_{\max } \sim 1 / \lambda$, with $\omega$ as the dimensional wave frequency. This relation enables us to estimate the length interval $(L)$ for maximum energy extraction as $L \lesssim c \omega / \lambda$. Considering the microwave range with $\lambda=0.01$ and $\omega \sim 10-100 \mathrm{GHz}$, one can approximately obtain $L \sim 1 \mathrm{~m}$ with acceleration gradients approximately lying in the range $1-100$ $\mathrm{MeV} / \mathrm{m}$.

To conclude, we have analyzed the cyclotronresonance laser accelerator in terms of a generalized Hamiltonian approach. The formulation is general in two basic senses; the dynamics of arbitrarily large laser fields is fully taken into account and the inclusion of effects connected with frequency mismatches and wave dispersion is allowed. By explicitly considering the microwave range, we have shown on theoretical grounds how an adequate adjustment of the mismatch factor can partially remove the limiting effects originated from wave dispersion on wave-particle energy exchanges. In the analyzed range some of the usual limits to energy exchange processes are not crucial, such as synchrotron emission and beam spread. Others, such as the bunching problem and energy spread, may become relevant if one takes not small enough values of $I_{0}$ or too large values of the mismatch as shown in the numerical simulations of Sec. IV B.

\section{ACKNOWLEDGMENTS}

This work was partially supported by Financiadora de Estudos e Projetos (FINEP) and Conselho Nacional de Desenvolvimento Científico e Tecnológico (CNPq), Brazil. Part of the numerical calculations were performed on the Cray YMP-2E computer of the Universidade Federal do Rio Grande do Sul Supercomputer Center.
[1] P. Sprangle, L. Vlahos, and C. M. Tang, IEEE Trans. Nucl. Sci. NS-30, 3177 (1983).

[2] C. S. Roberts and S. J. Buchsbaum, Phys. Rev. 135, 381 (1964).

[3] S. P. Kuo and G. Schmidt, J. Appl. Phys. 58, 3646 (1985).

[4] C. Chen, Phys. Fluids B 3, 2933 (1991); Phys. Rev. A 46, 6654 (1992).
[5] R. Shpitalnik, C. Cohen, F. Dothan, and L. Friedland, J. Appl. Phys. 70, 1101 (1991).

[6] R. Pakter, R. S. Schneider, and F. B. Rizzato, Phys. Rev. E 47, 3787 (1993).

[7] P. Sprangle, E. Esarey, and A. Ting, Phys. Rev. Lett. 64, 2011 (1990).

[8] A. Loeb and L. Friedland, Phys. Rev. A 33, 1828 (1986). 